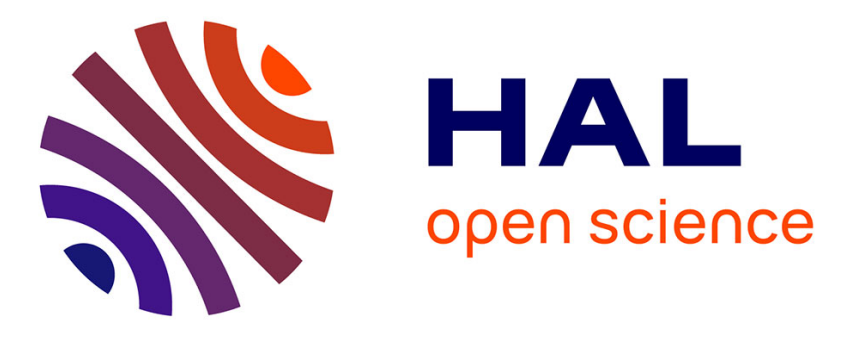

\title{
Integrated Digital Image Correlation considering gray level and blur variations: Application to distortion measurements of IR camera
}

\author{
Ali Charbal, John-Eric Dufour, Adrien Guery, François Hild, Stéphane Roux, \\ Ludovic Vincent, Martin Poncelet
}

\section{To cite this version:}

Ali Charbal, John-Eric Dufour, Adrien Guery, François Hild, Stéphane Roux, et al.. Integrated Digital Image Correlation considering gray level and blur variations: Application to distortion measurements of IR camera. Optics and Lasers in Engineering, 2016, 78, pp.75-85. 10.1016/j.optlaseng.2015.09.011 . hal-01303516

\section{HAL Id: hal-01303516 https://hal.science/hal-01303516}

Submitted on 18 Apr 2016

HAL is a multi-disciplinary open access archive for the deposit and dissemination of scientific research documents, whether they are published or not. The documents may come from teaching and research institutions in France or abroad, or from public or private research centers.
L'archive ouverte pluridisciplinaire HAL, est destinée au dépôt et à la diffusion de documents scientifiques de niveau recherche, publiés ou non, émanant des établissements d'enseignement et de recherche français ou étrangers, des laboratoires publics ou privés. 


\title{
Integrated Digital Image Correlation considering gray level and blur variations: Application to distortion measurements of IR camera
}

\author{
Ali Charbal, ", John-Eric Dufour, ${ }^{*}$ Adrien Guery, ${ }^{* 1}$ François Hild, \\ Stéphane Roux, ${ }^{*}$ Ludovic Vincent* and Martin Poncelet \\ *LMT, ENS Cachan / CNRS / Univ. Paris-Saclay, 61 avenue du Président Wilson \\ F-94235 Cachan cedex, France \\ \{charbal, dufour, guery, hild, poncelet, stephane.roux\}@lmt.ens-cachan.fr \\ *CEA, DEN, DMN, SRMA, F-91191 Gif sur Yvette cedex, France \\ ludovic.vincent@cea.fr
}

Corresponding author: François Hild

Email: francois.hild@1mt.ens-cachan.fr

Phone: +33147402192

Fax: +33147402240

\footnotetext{
${ }^{1}$ Present address: EdF - R\&D, Site des Renardières, avenue des Renardières - Ecuelles, F-77818 Moret-sur-Loing, France

adrien.guery@edf.fr -
} 


\title{
Integrated Digital Image Correlation considering gray level and blur variations: Application to distortion measurements of IR camera
}

\author{
The acquisition of images with different modalities may involve different \\ alterations with respect to an ideal model. Inhomogeneous brightness and \\ contrast, blur due to non-ideal focusing, distortions are common. It is proposed \\ herein to account for such effects for instance by registering a calibration target \\ image with an actual optical image to measure lens distortions. An Integrated \\ Digital Image Correlation (I-DIC) algorithm is proposed to account for the above \\ artifacts and the algorithm is detailed. The resolution and uncertainty of the \\ technique are first investigated on synthetic images, and then applied to the \\ measurement of distortions for infrared (IR) images. The procedure is shown to \\ reduce drastically the residual level assessing the validity of the image formation \\ model, but more importantly allowing for a much improved registration of \\ images.
}

Keywords: I-DIC; gray level conservation; blur; distortions; IR camera. 


\section{Introduction}

Digital Image Correlation (DIC) is more and more frequently used for applications dealing with full-field measurements [1]. The ever increasing quality and availability of imaging system allows for a widespread recourse to ever better quantitative measurements. Early on DIC was mostly used with visible light cameras [1]. This approach can be extended to images acquired with infrared cameras [2], scanning electron microscopes [3]-[5] confocal microscopy [6], atomic force microscopy [7-9] and X-ray tomography [10, 11]. In all cases, subpixel (subvoxel) displacement resolutions can be achieved.

In most DIC codes, the assumption of gray level conservation from the reference to the distorted images is considered. This assumption is often satisfied as the registered images are obtained with the same imaging system and lighting conditions. However this assumption is usually violated when the two images are obtained from different origins or imaging modalities (e.g., numerically generated reference images [4], [12], a hybrid stereovision system using one IR camera and one visible light camera to measure 3D surface displacements [13] and eventually 3D displacement fields and 2D temperature fields by extending the concept of IR image correlation [2]). For local approaches to DIC this conservation condition may be relaxed by various propositions [1] where offset and scale in lighting can be made irrelevant for the DIC analysis for each considered subset. However, as no additional constraints are set for the brightness and contrast corrections, it implies that two degrees of freedom for the subset registration are sacrificed (and generally not further exploited) at each measurement point. Although those spatial modulations of gray levels are not considered as a relevant and useful measurement, they involve a large number of unknowns that may compete with the kinematic degrees of freedom and hence alter the uncertainty especially for 
very small subset sizes. Alternatively, multimodal image registration may be achieved based on feature extraction and shape information [14], but this approach, which only uses a small part of the image information, is suited to elementary transformations such as rigid body motions.

Resorting to global DIC, where the entire image is considered at once, a limited spatial variability of these brightness or contrast corrections may easily be implemented [15] thereby allowing both for the consistent description of these artefacts, and yet, considering only a few parameters, these corrections have no detrimental effect on the uncertainty. Moreover, blur is generally ignored, yet imperfect lenses, limited depth of field, defocussing, are common features that may induce such effects. In the proposed approached, accounting for such blurring effect will be shown to be quite easy and rewarding.

Taking into account those artefacts is of general applicability to DIC, but appears to be especially necessary when dealing with images from different origin, or acquired by different image modalities. As a particular case, the assessment of optical distortions [16]-[19] can be achieved by using a DIC approach between a "reference model", i.e. a computer model printed with a high quality equipment, and images of this calibration target acquired with the camera to be analyzed. In this registration, one has to quantitatively describe how the image is formed, and hence, quantifying the gray levels involves a correspondence to be set, but inevitably, all kinds of artifacts such as inhomogeneous lighting or blurring effects are present. Blur is often modeled in the image processing literature as the convolution product between the sharp image and a filtering function that can be chosen as a Gaussian [20], or of a different type [21].

The paper is organized as follws. After a description of the proposed I-DIC approach to account for gray level variations and blurr, synthetic cases will be used to 
investigate the procedure sensitivity. Then, it will be applied to the distortion measurements of an IR camera. 


\section{Experimental procedure for the evaluation of distortions}

To estimate optical distortions, a calibration target (assumed to be perfectly known) is generally used in order to compare the measured positions of particular features of a numerical image [4], [12]. In the present case, for the IR camera considered in Section 5, the calibration target is a computer-generated pattern consisting of random dots (of known positions and sizes) [12] printed onto a stainless steel plate. The pattern is produced by first burnishing the entire surface, and then the layer is locally laser-removed thereby drawing the image negative (Figure 1). One may consider the numerical image, for such high quality printing, as "distortion-free."

(a)

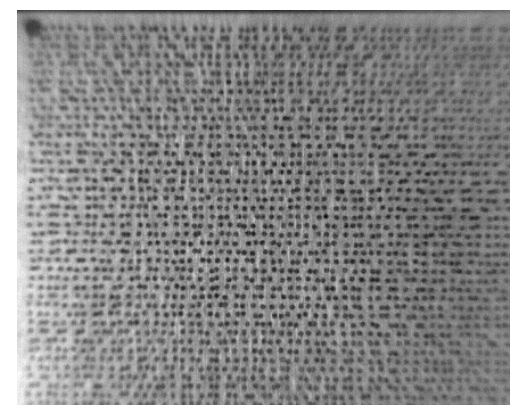

(b)

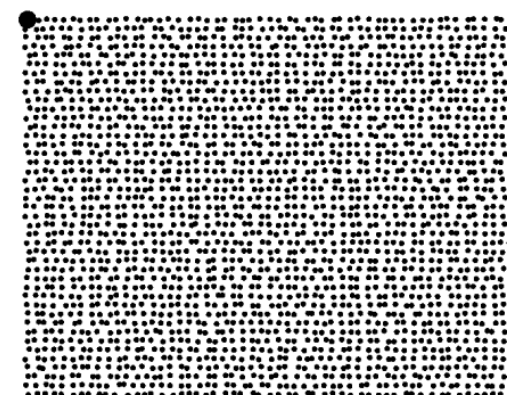

Figure 1: (a) Acquired (with IR camera) and (b) reference images of a calibration target

The IR camera used herein is a FLIR x6540sc, with a $512 \times 640$ pixel definition and 14-bit dynamic range. A 12-mm extension ring and a $50 \mathrm{~mm}$ lens are used so that the physical size of one pixel is $60 \mu \mathrm{m}$ at a working distance of $18 \mathrm{~cm}$. An exposure time of $1 \mathrm{~ms}$ is chosen. The camera is positioned in front of the calibration target and the optical axis is approximately normal to the sample surface. Images are shot at room temperature. As the speckle and background do not have similar emissivities they provide enough gray level contrast for DIC purposes. 


\section{I-DIC algorithm: gray level and blur corrections}

Figure 1 shows the differences between the distortion-free calibration target and the image acquired with the IR camera. The aim of the present paper is to propose a registration procedure that allows for the registration of those images at best.

\subsection{Gray level correspondence}

In this subsection, a general relationship between the numerical calibration target (i.e., gray level reference $f$ ) and experimental image (gray level distorted $g$ ) is proposed accounting for contrast, brightness and blur variations. This equation will then be implemented in an I-DIC code as a relaxation to the gray level conservation. It is chosen to correct the numerical (reference) image to avoid altering the raw experimental information.

The histogram of the experimental IR frame (shown in Figure 2(c)) allows a first rough determination of the gray level correspondence to be found between the reference and IR images. Figure 2(a-b) shows a zoom over the central part of the images of Figure 1, which is close to the optical axis where the optical distortions are minimal [22], and where the binary model has been constructed with the two gray levels determined from the histogram (Figure 2(c)) 
(a)

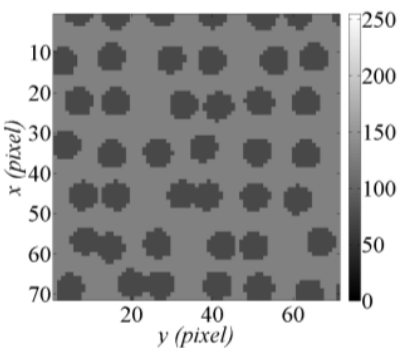

(b)

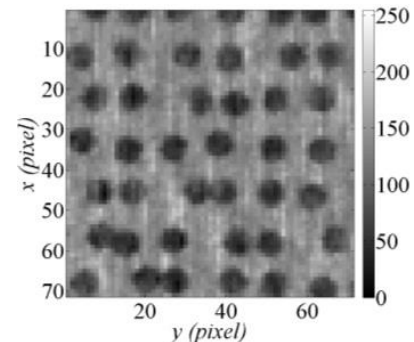

(c)

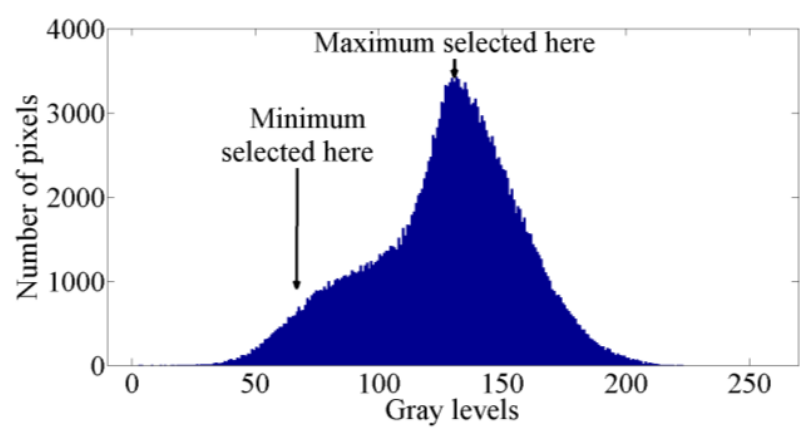

Figure 2: Regions of interest of the (a) numerical and (b) experimental images.

(c) Gray level histogram of the whole experimental image scaled down to a 8-bit dynamic range. The arrows show the two gray levels chosen for the binarization

After a rigid body motion correction and rescaling, these images should ideally coincide after a linear adjustment of gray levels. However this transformation is not the most faithful description of their relationship. In quantitative terms, the mean residual error, which is defined as the root mean square (RMS) of the gray level differences, is equal to $21 \%$ of the dynamic range of the picture. Similarly, the gray level histogram shows that the image is far from the bimodal gray level distribution that would be anticipated from such a simple linear correspondance.

Blur is described as a convolution between the ideal (sharp) image and a blurring kernel, $G$

$$
g(x, y)=(G * f)(x, y)
$$


The most elementary description of isotropic blur consists of choosing a Gaussian for the kernel. A finer description involves multiple Gaussians of different width. Let us introduce

$$
G_{i}(x, y)=\frac{1}{\sqrt{2 \pi} \sigma_{i}} \exp \left(-\frac{x^{2}+y^{2}}{2 \sigma_{i}^{2}}\right)
$$

with $\sigma_{i}=i$ with $i=1, \ldots, N$ is the width (in pixels), and $N$ the number of Gaussian functions. It is convenient to include in this series $G_{0}$, the limit of the Gaussian when $\sigma$ tends to 0 , that is a Dirac distribution, such that $G_{0} * f=f$. Hence, blur, brightness and contrast modulations are proposed to be described as

$$
g(\boldsymbol{x})=a+b_{i}\left(G_{i} * f\right)(\boldsymbol{x})
$$

It is noteworthy that taking into account $a$ and $b_{0}$ only corresponds to the classical gray level adjustment that is implicitly assumed using the ZNSSD (Zero-mean Normalized Sum of Squared Differences) criterion classically used in local DIC (see [1] for a detailed discussion of such criteria). Thus the introduction of non zero indices $i$ can be seen as an extension to global schemes. Moreover, the above expression is a linear combination of deterministic functions $G_{i} * f$ that can be pre-computed and thus Equation (3) is nothing but a linear regression.

Using three Gaussian kernels $i=0,1,2$ and a gray level offset $a$ (brightness adjustment) allows to match at best the target and IR images in their central part. Figure 3 shows the residuals obtained before and after histogram readjustment and with one Gaussian kernel $(N=1)$. The RMS residual when no gray level corrections (NGLC) are performed is equal to $48 \%$. It decreases to $11 \%$ of the dynamic range when a simple readjustment is performed with no blurring kernel $(N=0)$. It is further reduced 
to $10 \%$ after a blurring kernel is applied to the readjusted reference image. Resorting to a larger number of blurring kernels does not improve the result.

(a)

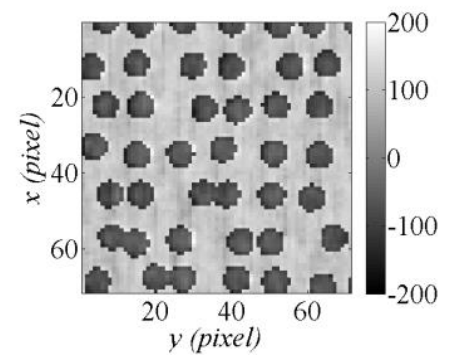

(b)

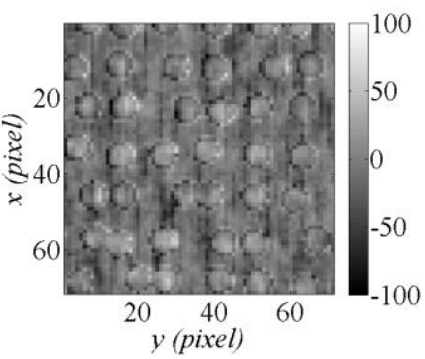

(c)

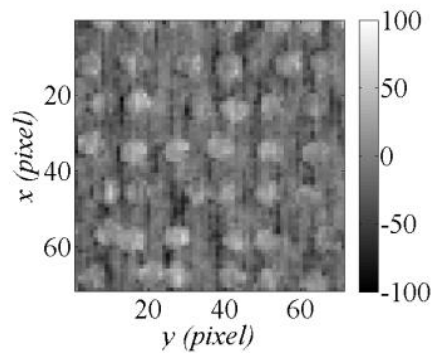

Figure 3: Gray level residuals obtained (a) before, (b) after histogram readjustment (and no blurring kernel, i.e. $N=0)$, and (c) one Gaussian kernel $(N=1)$

When considering the entire field of view, Figure 1 shows that such a form is not sufficient to account for the gray level transformation since the brightness varies spatially. One can also note that the blurring effect is more pronounced close to the image border. Hence, it is proposed to generalize the above form to

$$
g(\boldsymbol{x})=a(\boldsymbol{x})+\sum_{i=0}^{N} b_{i}(\boldsymbol{x})\left(G_{i} * f\right)(\boldsymbol{x})
$$

It is to be noted that without any further restriction on the variability of $a$ and $b$, the flexibility of such a transformation becomes detrimental to the determination of a kinematic field when the latter will be considered. To limit the variability of these parameters, a set of $M$ smooth functions $\varphi_{\mathrm{j}}$ is introduced and the parameters are sought under the following restricted form

$$
a(\boldsymbol{x})=\sum_{j=1}^{M} a_{j} \varphi_{j}(\boldsymbol{x}) \text { and } b_{i}(\boldsymbol{x})=\sum_{j=1}^{M} b_{i j} \varphi_{j}(\boldsymbol{x})
$$


Let us finally note that a non-uniform blur that would correspond to a spatially varying width $\sigma$ of the blurring kernel may, with an arbitrary quality, can be approximated by the above spatially modulated weights $b_{i}(\boldsymbol{x})$ of constant width convolutions. Hence the spatially varying gray level correspondence and blurring effects are captured by the set of parameters $\left(a_{j}, b_{i j}\right)$.

\subsection{I-DIC code}

In this subsection, the I-DIC algorithm is detailed. An equivalent formulation of the previously derived equation (4) relating both reference and distorted images is used, with the additional feature of accounting for a displacement field. In the sequel, the displacement field will be in fact a distortion field that describes the mispositioning of points from the reference target to the actual image. The sum of squared differences

$$
\eta=\sum_{R O I}\left(a(\boldsymbol{x})+\sum_{i=0}^{N} b_{i}(\boldsymbol{x})\left(G_{i} * f\right)(\boldsymbol{x})-g(\boldsymbol{x}+\boldsymbol{u}(\boldsymbol{x}))\right)^{2}
$$

is to be minimized over the region of interest (ROI). As mentioned above, the unknowns $\left(a, b_{i}\right)$ have a spatial variability constrained to a set of $M$ a priori chosen fields $\varphi_{j}$. Similarly, in the spirit of global DIC approaches [15], the displacement field is also expressed as a linear combination of $P$ chosen (vector) fields $\boldsymbol{\psi}_{k}$

$$
\boldsymbol{u}(\boldsymbol{x})=\sum_{k=1}^{P} u_{k} \boldsymbol{\psi}_{k}(\boldsymbol{x})
$$

The solution of this minimization is obtained thanks to a Newton-Raphson algorithm based on successive linearizations and corrections. At each iteration $n$ of the NewtonRaphson procedure, a linear problem is solved. It consists of $(N+2) M+P$ equations, where $M$ is the number of polynomial fields $\varphi_{j}$ used for gray level corrections, $(N+1)$ 
the number of Gaussian kernels (the additional 1 comes from $i=0$ ), and $P$ is the number of distortion fields $\boldsymbol{\psi}_{k}$.

The linear system (to be recomputed at each step $n$ ) reads

$$
\left[\boldsymbol{M}^{n}\right]\{\boldsymbol{\delta} \boldsymbol{u}\}=\left\{\boldsymbol{b}^{n}\right\}
$$

or

$$
\left[\sum_{R O I}\{\boldsymbol{m}(\boldsymbol{x})\}^{t}\{\boldsymbol{m}(\boldsymbol{x})\}\right]\{\boldsymbol{\delta u}\}=\left\{\sum_{\boldsymbol{R O I}}\{\boldsymbol{m}(\boldsymbol{x})\}^{t}\left(f^{n}-g^{n}\right)(\boldsymbol{x})\right\}
$$

where all the corrections to the unknown amplitudes are gathered into a single vector $\{\boldsymbol{\delta} \boldsymbol{u}\}$

$$
\{\boldsymbol{\delta} \boldsymbol{u}\}^{\boldsymbol{T}}=\left\{\delta a_{j}, \delta b_{i j}, \delta u_{k}\right\}
$$

vector $\{\boldsymbol{m}\}$ reads

$$
\{\boldsymbol{m}(\boldsymbol{x})\}=\left\{\varphi_{j}(\boldsymbol{x}), \varphi_{j}(\boldsymbol{x})\left(G_{i} * f\right)(\boldsymbol{x}), \boldsymbol{\psi}_{k}(\boldsymbol{x}) \cdot \boldsymbol{\nabla} g^{\boldsymbol{n}}(\boldsymbol{x})\right\}
$$

and $g^{n}(\boldsymbol{x})$ is the deformed image corrected by the current determination of the displacement

$$
g^{n}(\boldsymbol{x})=g\left(\boldsymbol{x}+\boldsymbol{u}^{n}(\boldsymbol{x})\right)
$$

where $\boldsymbol{u}^{n}(\boldsymbol{x})$ is the current estimate of the displacement field. The updating step of $g$ constitutes the non-linear part of the problem. The current corrected reference is expressed as

$$
f^{n}(\boldsymbol{x})=a^{n}(\boldsymbol{x})+\sum_{i=0}^{N} b_{i}^{n}(\boldsymbol{x})\left(G_{i} * f\right)(\boldsymbol{x})
$$


where $a^{n}(\boldsymbol{x})$ and $b_{i}^{n}(\boldsymbol{x})$ are the current estimates of the brightness and contrast correction fields. The iterative algorithm is driven up to the stage where the incremental changes in the gray level or displacement parameters become lower than than a chosen threshold $\left(10^{-5}\right.$ in the present case). Table 1 shows the set of displacement fields $\boldsymbol{\psi}_{k}$ used in this study. They follow the standard classification of the main components of optical distortions [22], [23] as discussed in Ref. [12]. Distortion fields are then expressed in coordinates $\left(x_{r}, y_{r}\right)$ whose origin is the optical axis while the additional fields are expressed in terms of the image coordinates $(x, y)[12]$. Let $L$ denote a characteristic length (e.g., width or height of the image expressed in pixels). The dimensionless coordinates are then defined as $(X=x / L, Y=y / L)$ and $\left(X_{r}=x_{r} / \mathrm{L}\right.$ ,$\left.Y_{r}=y_{r} / \mathrm{L}\right)$ to ensure a good conditioning of the linear systems to be solved [12]. 
Table 1: Kinematic fields used for distortion estimation in dimensionless coordinates [12]

\begin{tabular}{|c|c|}
\hline Designation & Interpolation fields \\
\hline directions & $\psi_{1 x}=1$ and $\psi_{1 y}=0$ \\
\hline Linear transformation in X & $\psi_{2 x}=0$ and $\psi_{2 y}=1$ \\
\hline Linear transformation in $\mathrm{Y}$ & $\psi_{3 x}=X_{r}$ and $\psi_{3 y}=0$ \\
\hline Decentering along $\mathrm{X}$ & $\psi_{4 x}=0$ and $\psi_{4 y}=X_{r}$ \\
\hline Decentering along $\mathrm{Y}$ & $\psi_{5 x}=Y_{r}$ and $\psi_{5 y}=0$ \\
\hline Prismatic along X & $\psi_{6 x}=0$ and $\psi_{6 y}=Y_{r}$ \\
\hline Prismatic along Y & $\psi_{7 x}=\left(-3 X_{r}{ }^{2}+Y_{r}{ }^{2}\right)$ and $\psi_{7 y}=2 X_{r} Y_{r}$ \\
\hline Radial distortion & $\psi_{8 x}=2 X_{r} Y_{r}$ and $\psi_{8 y}=\left(-3 X_{r}{ }^{2}+Y_{r}{ }^{2}\right)$ \\
\hline & $\psi_{9 x}=\left(X_{r}{ }^{2}+Y_{r}{ }^{2}\right)$ and $\psi_{9 y}=0$ \\
\hline & $\psi_{10 x}=0$ and $\psi_{10 y}=\left(X_{r}{ }^{2}+Y_{r}{ }^{2}\right)$ \\
\hline
\end{tabular}

The brightness, contrast and blur corrections are modulated by fields set to low order polynomials up to degree 2 . In the following, various combinations as detailed in Table 2 will be used and probed. 
Table 2: Interpolation fields used for gray level corrections and blurring effects

\begin{tabular}{|c|c|c|}
\hline Designation & $M$ & Interpolation fields \\
\hline Constant & 1 & $\varphi_{1}=1$ \\
\hline Linear & 3 & $\varphi_{2}=X, \varphi_{3}=Y$ \\
\hline Bilinear & 4 & $\varphi_{4}=X Y$ \\
\hline Order 2 & 6 & $\varphi_{5}=X^{2}, \varphi_{6}=Y^{2}$ \\
\hline
\end{tabular}

\section{Artificial test cases}

In order to validate the I-DIC code, numerical test cases are analyzed in which distortions and gray level variations are artificially applied. The application of distortion fields is performed by moving the dot center position of a given displacement field parameterized by the corresponding amplitude. The size (and shape) of the dots is not altered. However, as small strains will be considered, and because of blur that is present in the image, this omission has no influence. Based on prior tests performed on an IR camera with an arbitrary distortion basis, a set of representative values has been selected to create this synthetic test. Table 3 summarizes the values of non-zero parameters chosen for the test. They involve only rigid body motions, radial and prismatic modes. The decentering is determined through the distortion center position $(\mathrm{Xo}, \mathrm{Yo})$. The images are interpolated with linear functions and encoded in 8-bit.

\subsection{Distortion analysis}

A first test case is used where only distortions are applied. Both reference and distorted images have the same gray level distributions. The images have a definition of 
$1000 \times 1000$ pixels. Table 3 lists the prescribed and measured distortion parameters on binary images.

Table 3: Prescribed and measured distortion parameters (expressed in pixels)

\begin{tabular}{|c|c|c|}
\hline Designation & Prescribed & I-DIC \\
\hline Rigid body motions along X & 0.200 & 0.206 \\
\hline Rigid body motions along Y & 0.220 & 0.225 \\
\hline Radial along X and Y & 5.66 & 5.68 \\
\hline Prismatic along X & 0.10 & 0.06 \\
\hline Prismatic along Y & -1.55 & -1.54 \\
\hline Xo position & 420 & 419 \\
\hline Yo position & 350 & 354 \\
\hline
\end{tabular}

The gap between the prescribed and determined parameters is very small since the L2norm of the translation or distortion parameters difference (first two lines or three following lines of Table 3 ) is $5.5 \times 10^{-3}$ pixel for rigid body motions and $2.6 \times 10^{-2}$ pixel for distortion parameters. 


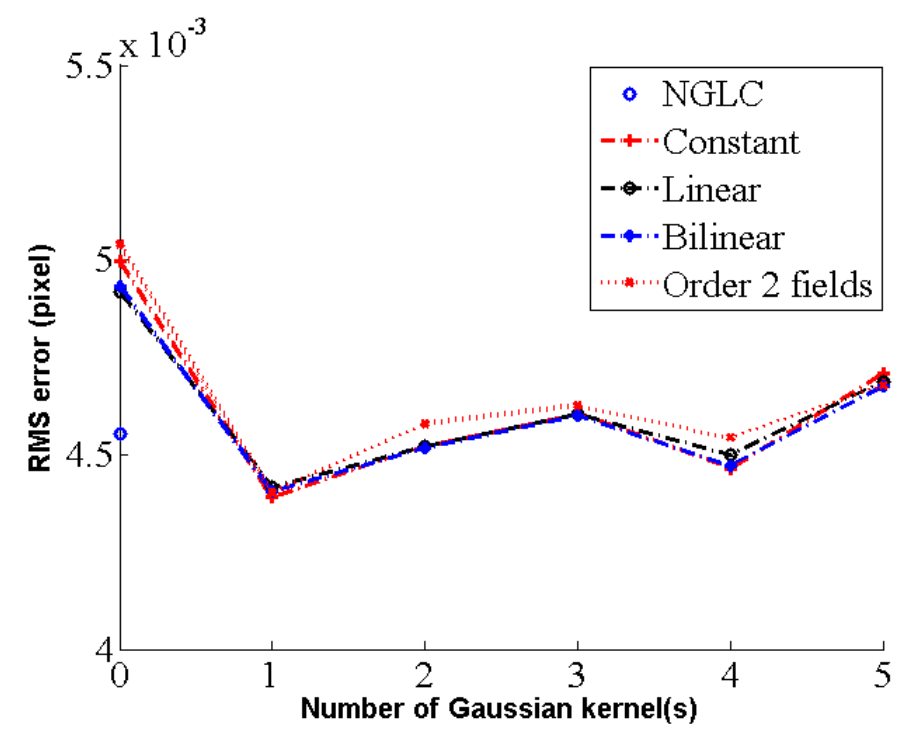

Figure 4: RMS difference between the prescribed displacement field and the measured results for different settings of I-DIC (binary distorted image)

When the I-DIC code is run without any gray level correction the RMS level reaches $4.7 \cdot 10^{-3}$ pixel (Figure 4). This result shows that very small differences occur (between the different strategies of gray level corrections), thereby fully validating the I-DIC code. The gray level residuals (see Figure 5) decrease as soon as blurring functions are added. The dimensionless RMS varies from $12 \%$ with no gray level correction (NGLC) to $11.4 \%$ for any tested number $M$ of supporting fields for brightness and contrast corrections. As soon as one blurring kernel is added the mean residual drops to $8.8 \%$ of the dynamic range and decreases only slightly with the addition of more blurring kernels. 


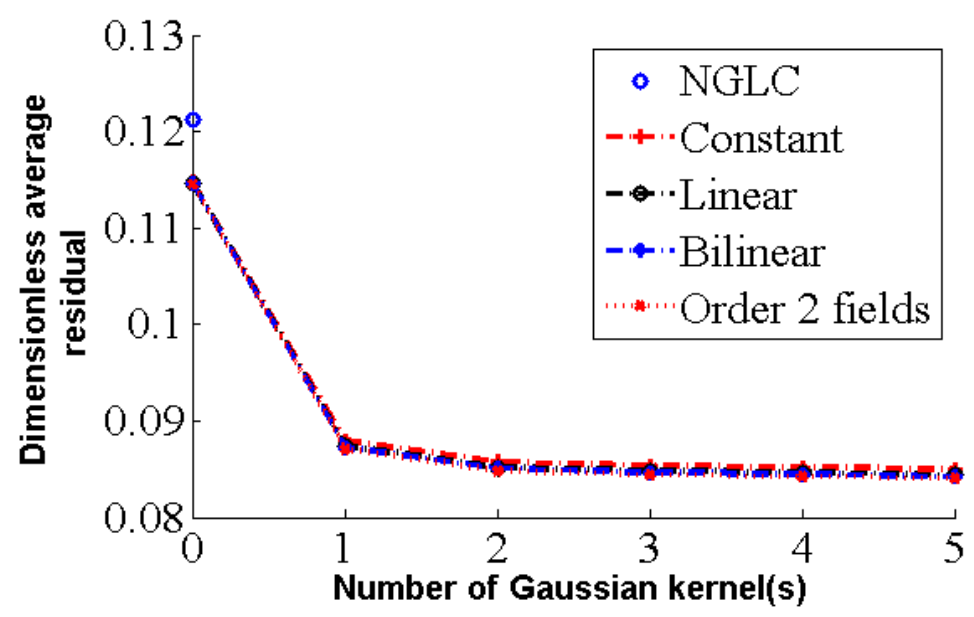

Figure 5: Nominal DIC residual error with the different types of gray level and blur corrections (binary distorted image)

Because the synthetic image is generated using a sub-pixel interpolation scheme, the boundary of the dots is no longer sharp. Therefore blurring the reference image, produces a smoother image and hence a more faithful interpolation. Nonetheless the values of the gray level errors are still high ( $8 \%$ of the dynamic range) while registering binary images. To reduce the residual levels, it is possible to blur not only the reference but also the distorted images, and to perform similar calculations. The results are very similar in terms of the sought parameters and displacement field. The RMS difference between the results obtained with two binary or two blurred images is $1.2 \cdot 10^{-4}$ pixel. The gray level residuals are significantly reduced (namely from $8 \%$ to $1.9 \%$ for the dimensionless average residuals in the previous case). It is important to comment on the apparent paradox of the usefulness of blurring herein. Blurring an image induces some loss of information on a general ground. However, image registration is only based on image features that are shared by the different images. The purpose of blurring then is to remove a part of the signal that is present in one single image in order to isolate only shared features. Thus, for DIC purposes (and not for other usages), the only "lost" 
information is the one that would have fooled registration and induced error.

\subsection{Gray level variations}

In the following case (Figure 6) the distorted image has gray level variations along the $y$ axis following a sine like function

$$
a(x, y)=78 \cdot \sin \left(\frac{2 \pi y}{1500}\right)+78
$$

when $a$ is expressed in gray levels, and $y$ in pixels. The image is also blurred uniformly with 2 blurring kernels and corrupted by Gaussian white noise (of standard deviation equal to 5.8 gray levels).

(a)
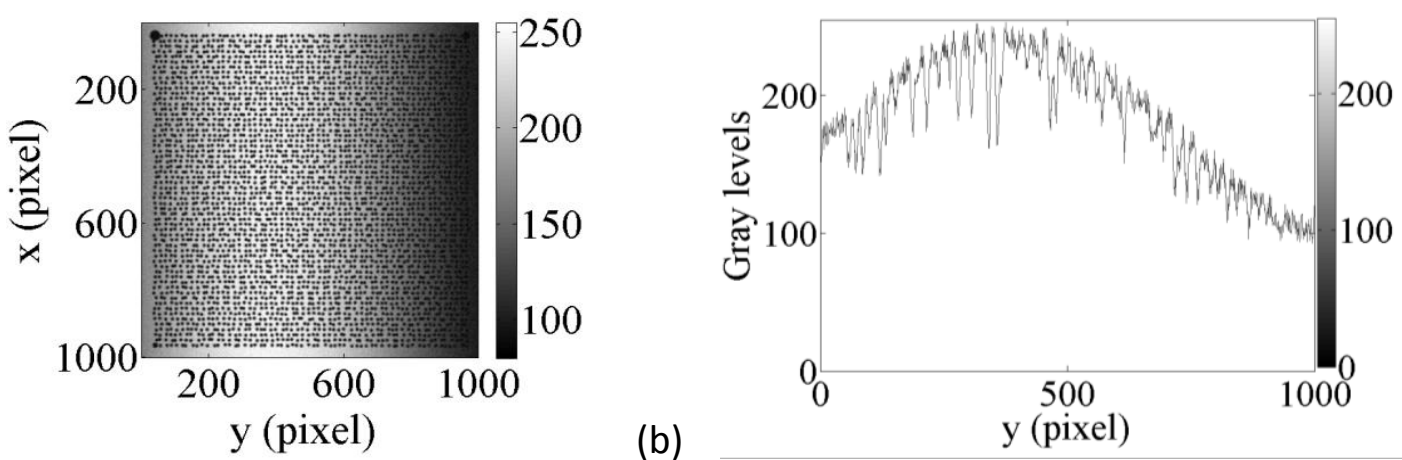

Figure 6: (a) Distorted image biased by sine-like gray level variations along the $y$ direction, blur and noise and (b) its gray level profile

This critical case allows the code to be tested but also the error to be assessed when no gray level readjustments are performed. Similar distortion parameters as in the previous case are then applied in addition to the other sources of error. The choice of using sinusoidal gray level variations is to check the robustness of the proposed approach since the present I-DIC code uses polynomial fields. Adding noise will permit to be as close as possible to real cases where the acquisition is corrupted by noise. 
The first analysis is performed on the determination of the center position $X o$ (equivalent results are obtained for the position $Y o$ ) prescribed to be 410 pixels. This analysis will highlight which gray level correction basis provides the best estimates of this parameter (Figure 7). NGLC-DIC provides a gap of 35 pixels on the determination of the Xo position. If gray level corrections are introduced with a uniform field $(M=1)$ the gap reduces to 17 pixels. If linear or bilinear bases $(M=3,4)$ are used, the determined Xo position is 405 pixels (instead of 410 pixels) but drifts away to 400 pixels when 4 blurring kernels are added. When fields of order 2 are added $(M=6)$ the determined positions are very close to the prescribed ones (i.e., the gap is less than 5 pixels after one blurring kernel is added, and does not change when more blurring kernels are considered).

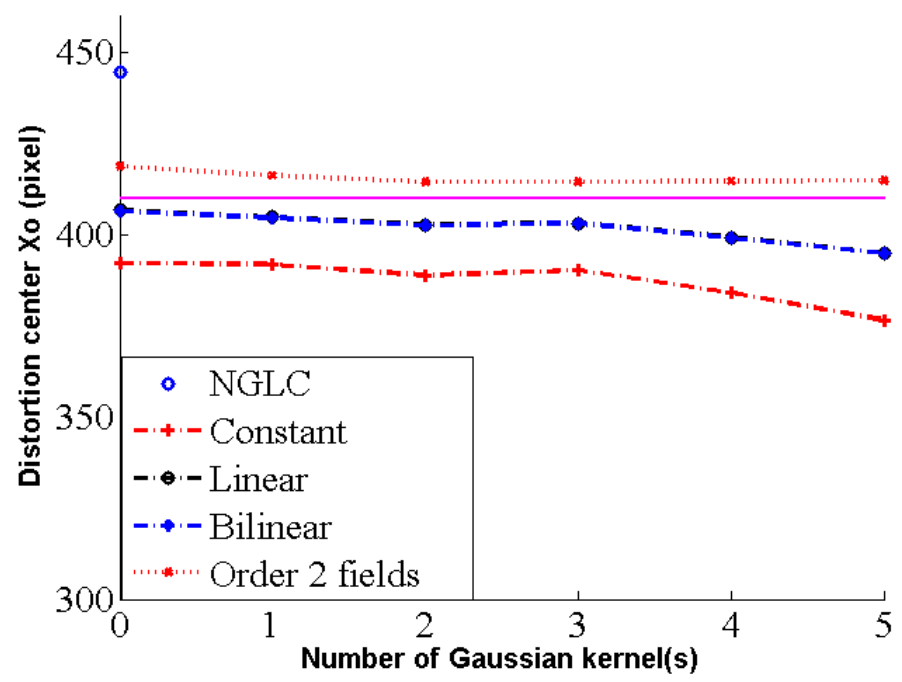

Figure 7: Distortion centre position $X o$ (pixel) for different gray level and blur correction functions (biased and distorted image)

The same comparison is performed on the radial distortion parameter in Figure 8. When NGLC is considered the error on the radial parameter determination is as high as 1.75 pixel. The constant, linear and bilinear fields $(M=1-4)$ allow to approach the 
parameters reducing the error down to $0.7-0.4$ pixel, respectively. It tends to increase above 3 Gaussian kernels up to $0.9-0.6$ pixel. As soon as fields of order $2(M=6)$ are used the gap is reduced 0.1 pixel for any number of blurring kernels. This trend is comparable to the accuracy observed in the binary case. The parameter determination is not very sensitive to blur corrections when they are added. The order 2 fields used for gray level corrections provide a better estimate of the radial parameter. This is also illustrated by the center position, which is dependent on both prismatic and radial parameters.

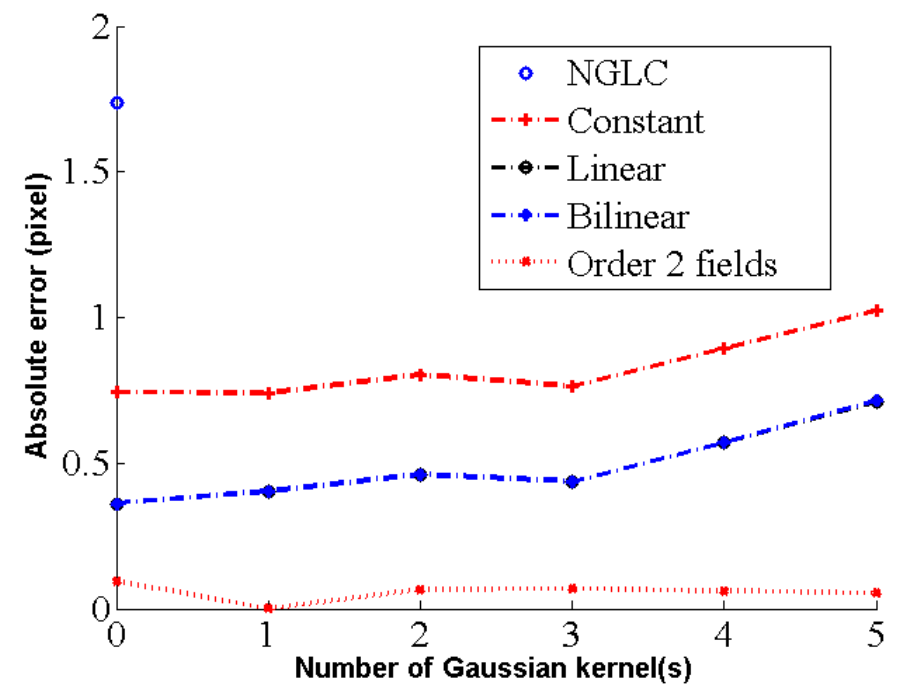

Figure 8: Absolute error on the radial parameter (biased and distorted image) for different gray level corrections and blurring kernels

The displacement fields are analyzed for different corrections. The RMS difference between the measured and the prescribed fields are plotted in Figure 9. With a standard I-DIC code the RMS level amounts to $1.5 \times 10^{-1}$ pixel, which is a high value given the fact that only distortion parameters are determined [12]. If constant fields are used the RMS error decreases to $6.5 \times 10^{-2}$ pixel, and increases to higher 
values $\left(9.0 \times 10^{-2}\right.$ pixel $)$ for more than 3 blurring kernels. The linear and bilinear fields provide RMS levels of $5.7 \times 10^{-2}$ pixel and again increase to $9.0 \times 10^{-2}$ pixel for more than 3 Gaussian kernels. Using higher order polynomial fields decreases the RMS error to $2.0 \times 10^{-2}$ pixel and $6 \times 10^{-3}$ pixel (these levels have to be compared with the previous results on binary images). The blurring fields do not affect much the RMS error for more than two kernels.

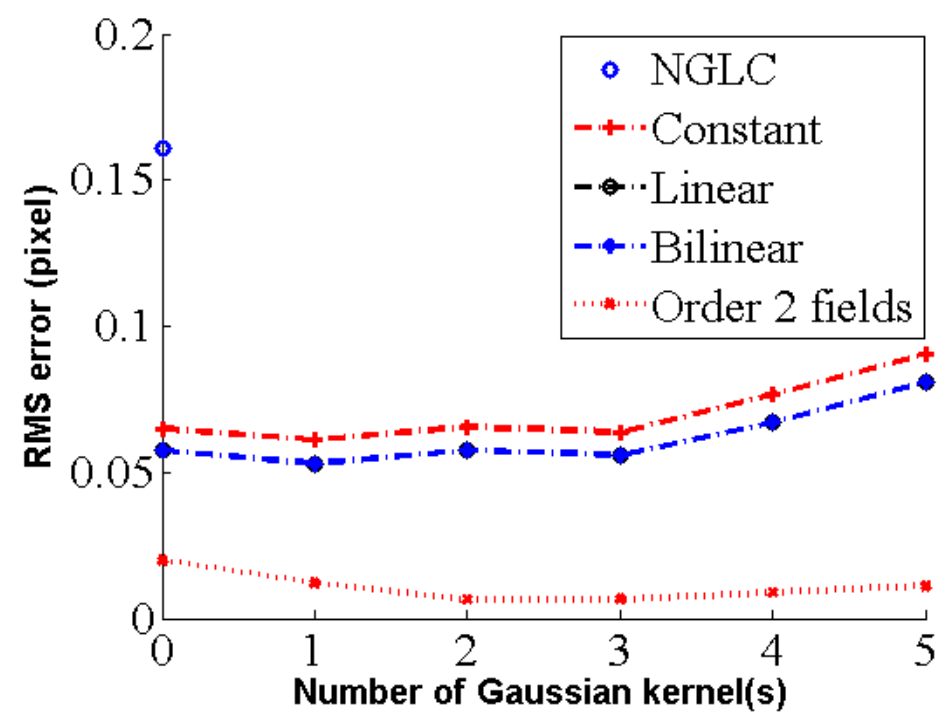

Figure 9: RMS difference between the prescribed displacement field and the measured results for different settings of I-DIC (biased and distorted image)

The gray level errors are a way of characterizing the quality of the measured displacement field when the latter is a priori unknown. When NGLC is applied the RMS residual is $38 \%$ of the dynamic range. It then reduces to $22.6 \%$ with a constant interpolation field and when considering blurring kernels it further decreases to $22 \%$. For linear and bilinear interpolations the mean errors are lowered to $18.5 \%$ and to $16 \%$ when blurring functions are added. The gray level residual for order 2 fields is equal to 
$10 \%$ before adding Gaussian kernels and $4 \%$ thereafter. It is clearly advantageous for the gray level residuals to add blurring kernels.

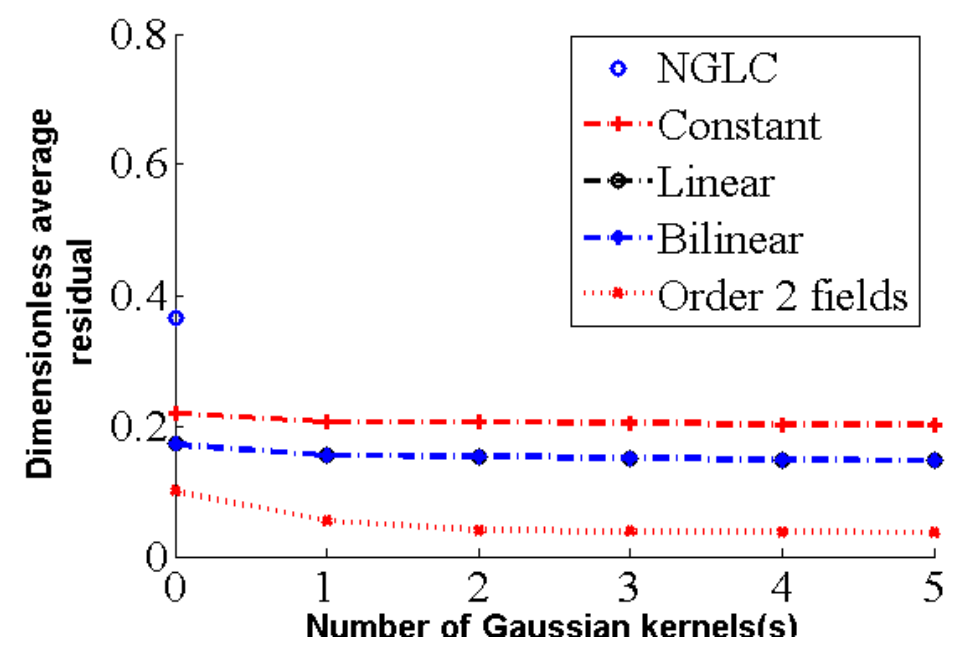

Figure 10: DIC residual for different types of gray level and blur corrections (biased and distorted image)

Thus it appears as very important to include shape functions of order 2 for the gray level modulations. The number of blurring kernels past the first or second ones play a more modest role. If not enough shape functions are considered to account precisely for inhomogeneous illumination, further degrees of freedom such as an increase in the number of blurring kernel appears detrimental to the residual level. This effect shows that when images cannot be perfectly registered, additional inadequate degrees of freedom that may have expected to be neutral are in fact prejudicial.

The choice of field order and number of blurring kernels may depend on the analyzed images. Even if the user introduces more degrees of freedom than needed the I-DIC code provides good results as proven by the test case on binary images. For instance the IR image (Figure 1) shows gray level variations and blur. The edges are more prone to such variations than the center, this is well approached by fields of order 
2 and two blurring kernels should be sufficient. This suggestion is tested in the next part.

\section{Application to IR image}

Having validated the whole procedure on artificial test cases, the I-DIC analysis is now applied to actual IR pictures. The acquired images are compared to binary references having gray level amplitudes ranging from 0 to 255 (i.e., 8-bit digitization). Comparisons with a global DIC calculation with no a priori knowledge of the displacement fields will also be performed. This will be an additional validation step of I-DIC.

The gray level corrections are illustrated in Figure 11 starting from the raw reference image when correction for gray level differences and blur are performed. The first image corresponds to the binary reference (Figure 11(a)). The second is a corrected reference image when a second order field is chosen and no blur is accounted for (Figure 11(b)). The third image is accounting for gray level variations and blur (with 2 blurring kernels, see Figure 11(c)). The last image is the experimental acquisition (Figure $11(d)$ ). 


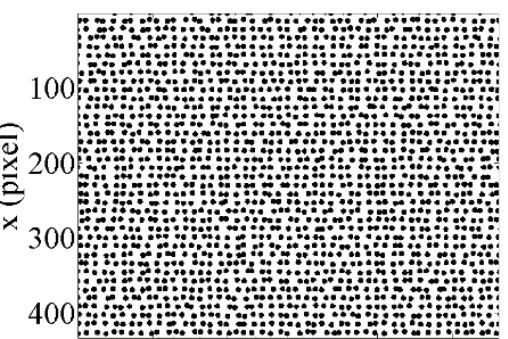

(a)

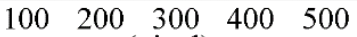

(b)
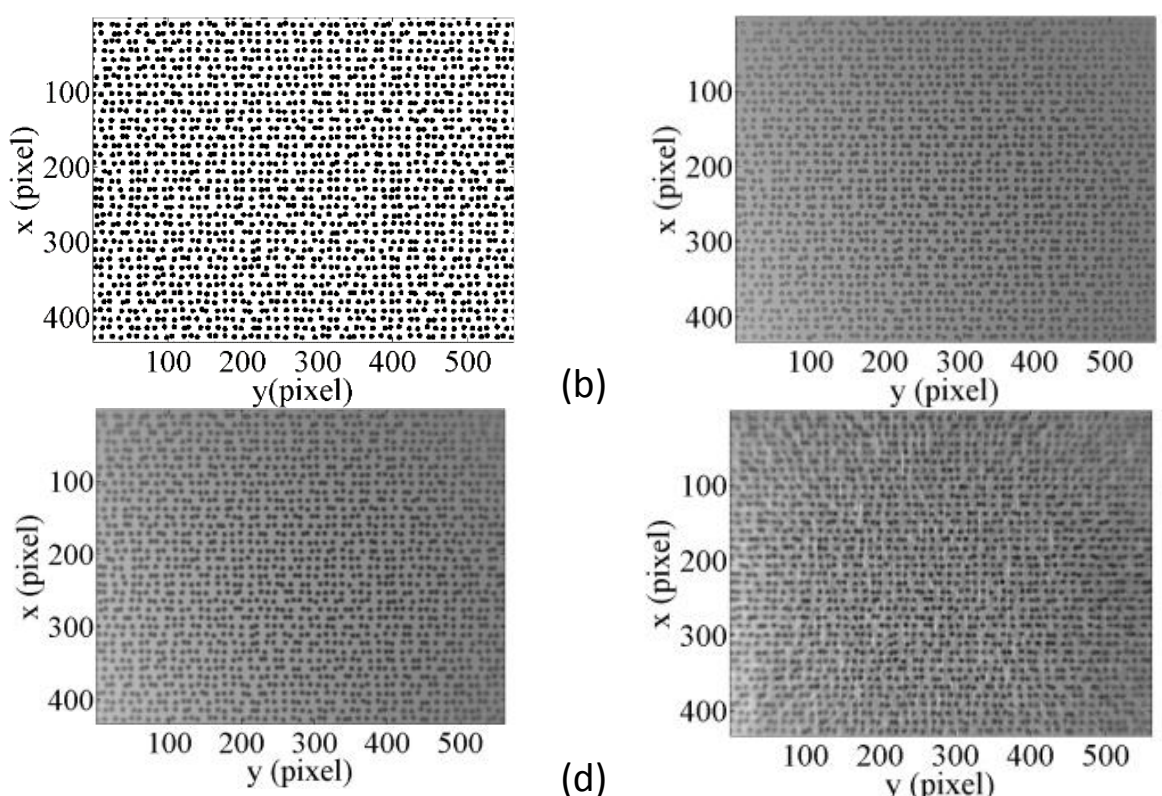

(d)

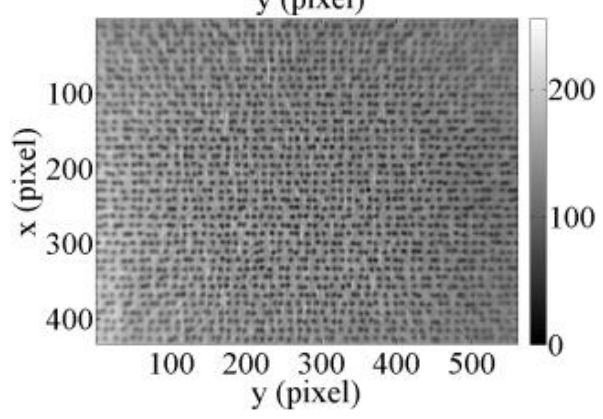

Figure 11: Reference image (a) when no corrections are performed, (b) with corrections using order 2 fields with no blurring kernels and (c) with blurring kernels.

(d) Experimental IR picture

As more corrections are added, the global aspect of the reference image looks more similar to the one acquired by the IR camera. These results are analyzed with the gray level histograms of each presented image. A large difference between the histograms of the binary and the experimental image is observed when Figure 12(a) is compared to Figure 12(d). A clear improvement is observed by considering only gray level corrections (although with order 2 spatial modulations, Figure 12(b)) and the histogram is approaching the experimental one when blurring fields are added (Figure 12(c)). 
(a)

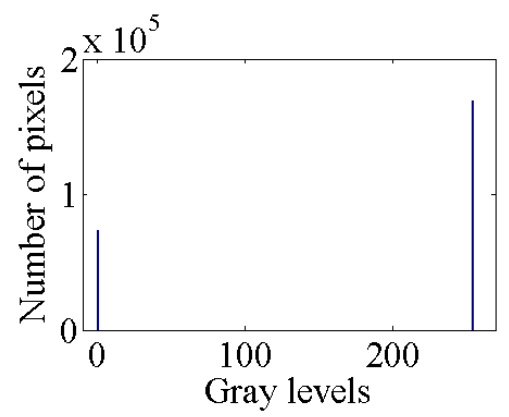

(c)

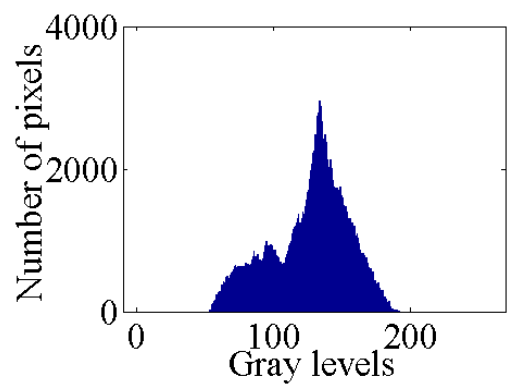

(b)

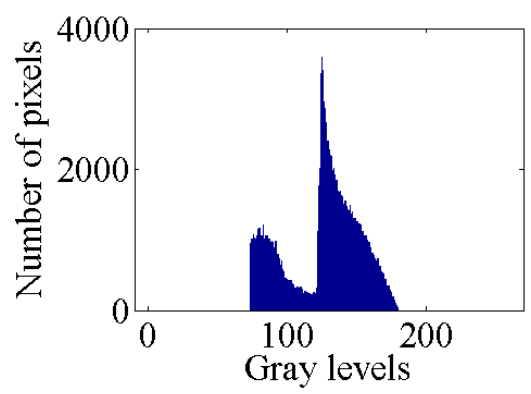

(d)

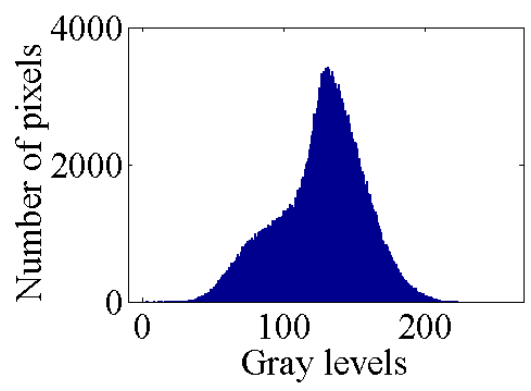

Figure 12: Gray level histograms corresponding to the four pictures shown in Figure 11

These observations are also supported by considering the gray level residual maps. Figure 13(a-b) correspond to an I-DIC analysis in which the gray level correction uses a constant field without and with blurring corrections (with 2 Gaussian kernels), respectively. The blurring corrections provide more homogeneous residual maps. The results of Figure 13(c-d) are obtained when considering gray level corrections with second order fields without and with blurring kernels, respectively. Increasing the order of the fields decreases the gray level residual map (see Figure 13 (a-c)). Adding blurring kernels yields a more homogeneous residual map. 
(a)

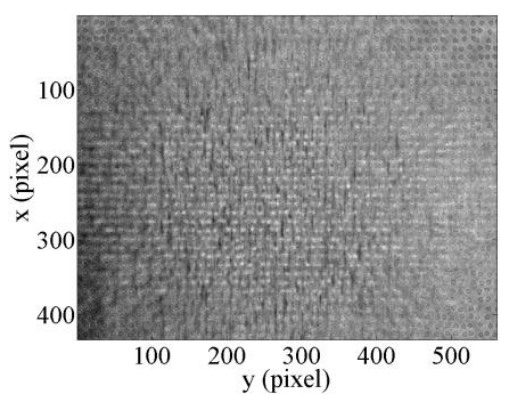

(c)

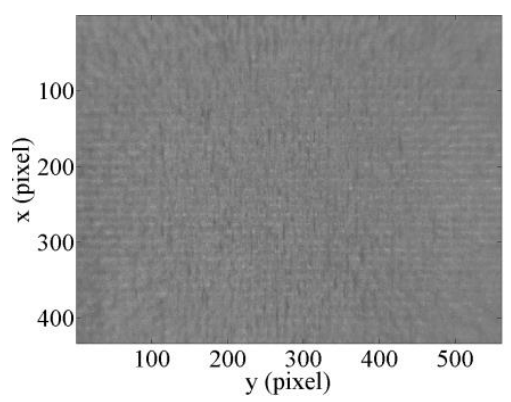

(b)

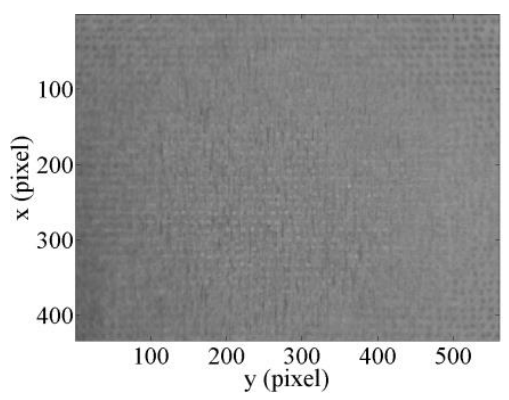

(d)

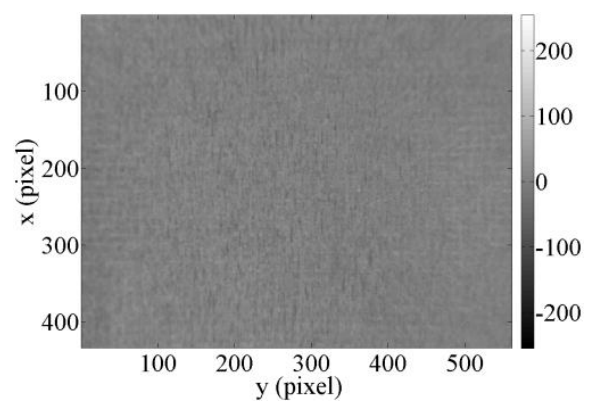

Figure 13: Gray level residuals for distortion estimations of the IR camera when gray level corrections are performed with (a) constant fields, and (b) 2 blurring kernels, (c) second order fields and (d) with blurring kernels

The mean dimensionless residual is plotted for the different sets of gray level and blur readjustments in Figure 14. The method with NGLC is converging with a final dimensionless residual of $38 \%$. Once corrections are considered, the residuals are reduced with the order of the polynomial field to the correct gray levels. The mean dimensionless residual is equal to 8 and $7 \%$ of the dynamic range respectively for constant fields without and with blurring kernels (Figure 13 (a-b)). They are significantly reduced to reach levels of the order of $6 \%$ and $4 \%$ for second order fields without and with blur corrections (Figure 13 (c-d)). The addition of one blurring kernel is sufficient to reach quasi uniform correlation residuals. 


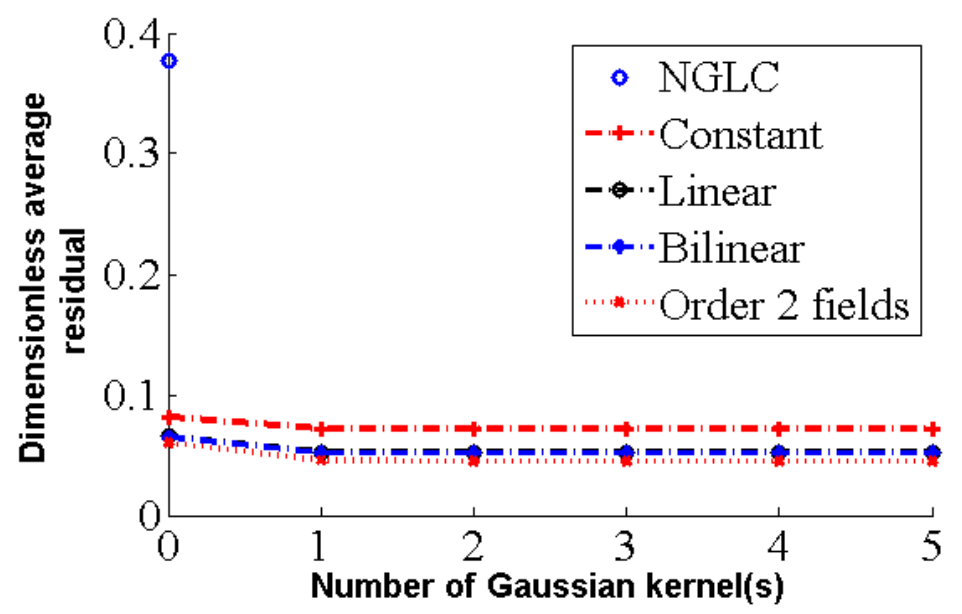

Figure 14: Dimensionless gray level residual with different types of gray level and blur corrections for the IR image

It is worth noting that the correlation residuals are still greater than those only associated with acquisition noise $(0.4 \%)$, which is an indication of a model error of the distortions. In the proposed method, only first order expansions of distortions are considered, which seems to be sufficient to consider most of the effects. As discussed in Ref. [12], nonparametric bases (e.g., cubic B-splines of different orders) can also be used to account for distortions, leading to lower gray level residuals.

It has been shown that the gray level corrections are well approached by I-DIC through the histograms or residual error analyses. The latter is representative of the quality of the registration in terms of gray level conservation but also on the displacement field determination. Therefore to test the validity of the gray level corrections of the I-DIC approach, an additional check is performed by using a general purpose DIC code with no a priori knowledge on the kinematic fields. The code used herein is based on a regularized finite element discretization composed of 3-noded triangles (i.e., RT3-DIC [24], which was already used to analyze the distortions of SEM pictures). The T3 element size is equal to 5 pixels with a regularization length of 
128 pixels. The results are compared to the best results provided by the I-DIC approach (i.e., with the lowest gray level residual).

The measured displacement fields are very close in terms of shape and level (see Figure 15). Even though the RT3-DIC code only corrects for brightness and contrast deviations using constant fields, the dimensionless gray level error is equal to $34 \%$. If no regularization were used RT3-DIC would not converge since the element size is too small. The measured results are similar when a regularisation length of 256 or 64 pixels is used. The RMS difference between the two sets of regularization lengths is about 0.01 pixel, which is very low. Using one regularisation length or another will not affect the final conclusion concerning the I-DIC results. Therefore only the kinematic fields obtained with a regularization length of 128 pixels are compared to the I-DIC results obtained with different gray level corrections. 
(a)

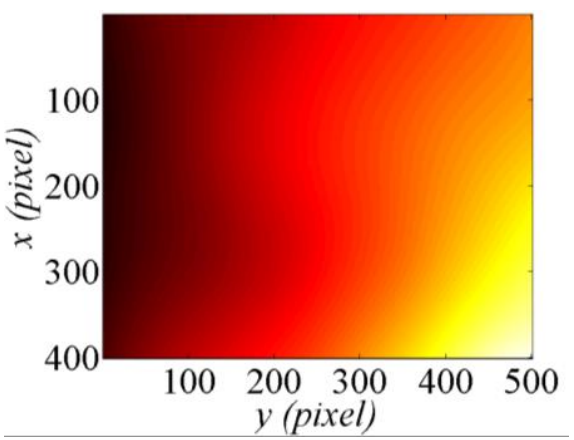

(c)

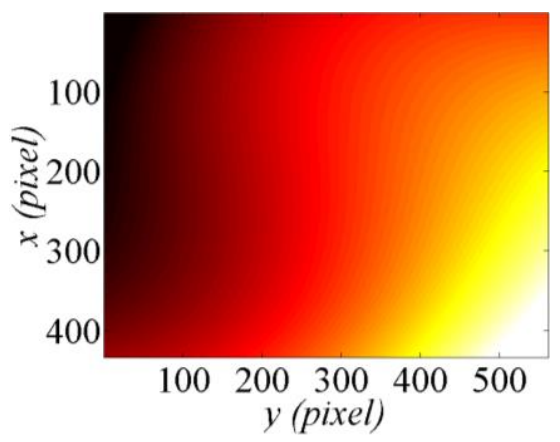

(b)

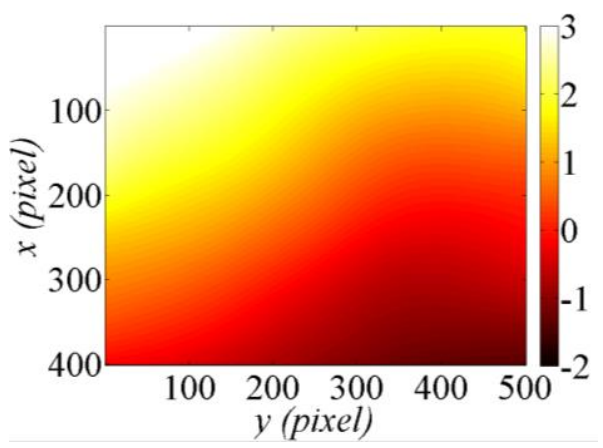

(d)

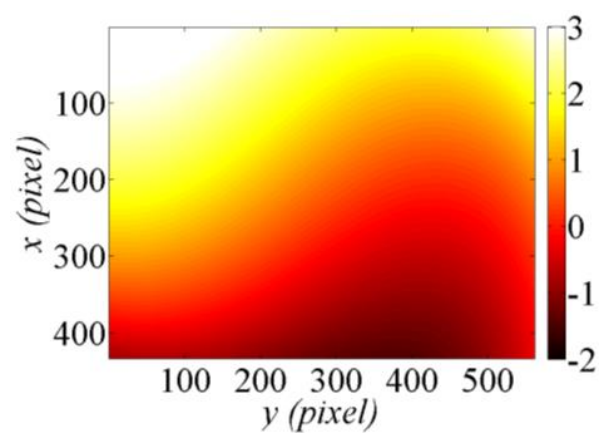

Figure 15: Measured displacement fields with RT3-DIC along (a) $y$ and (b) $x$ directions, and I-DIC approach with second order fields and 2 Gaussian kernels along (c) $y$ and (d) $x$ directions for the IR image

The RMS difference between the measured displacement fields via RT3-DIC and the different sets of gray level and blur corrections in I-DIC are plotted in Figure 16. When no gray level corrections are applied in the I-DIC method, the RMS difference is equal to 0.18 pixel. It decreases to 0.13 pixel for constant, linear and bilinear interpolation fields. For the order 2 fields of gray level interpolation in the IDIC method, the RMS difference decreases to 0.12 pixel. Adding blur slightly increases the RMS difference. From constant to bilinear fields, similar results are obtained in terms of RMS difference on the displacement fields. Whereas when NGLC is applied or second order fields are used the RMS difference varies compared with the I-DIC result obtained with constant fields. This proves that correcting for gray level variations is influencial on the measured displacement fields. The fact that RMS difference between 
RT3-DIC and I-DIC is mostly insensitive to gray level corrections and blurring kernels is an indication that there is another cause at play. It is believed that most of the difference is due to errrors associated with the distortion model. A similar observation was made when analyzing regular cameras [12] and SEM pictures [4].

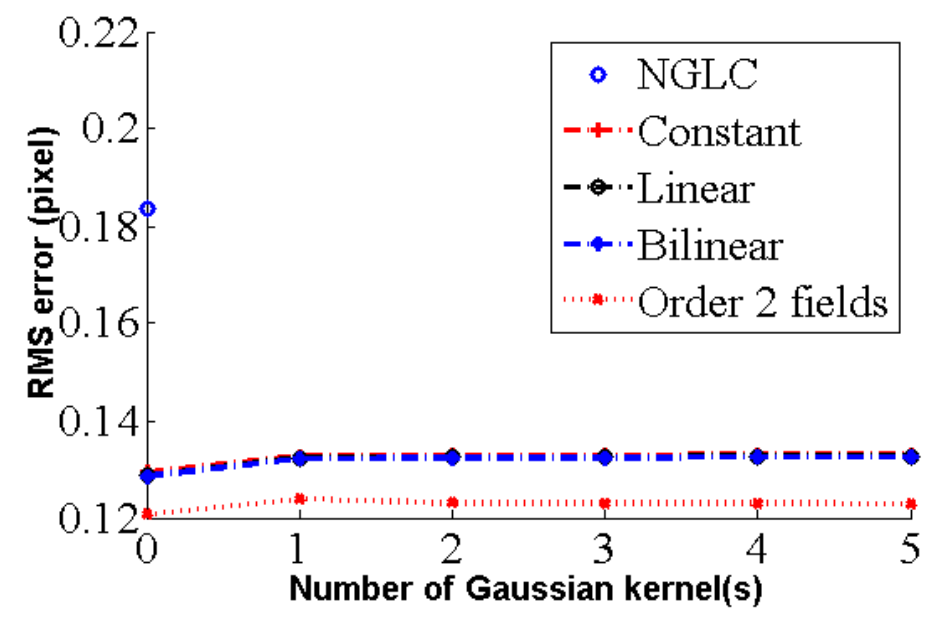

Figure 16: RMS of the difference between the displacement fields obtained for different set of the I-DIC and the RT3 approaches (IR images)

According to the previous test cases, fields of order 2 with one or two blurring kernels provided the best estimates of the sought parameters. This is well correlated with the gray level residuals. The DIC residual analysis has shown that the best results are provided by order 2 fields with one or two blurring kernel(s) for the analyzed IR image. The following discussions on the distortion fields will be conducted with this last result. It is worth mentioning that the RMS difference in terms of the measured distortion fields or parameters between a constant field and order two fields is as small as 0.02 pixel.

The distortion fields along the $x$ and $y$ directions are reported in Figure 17. The results show that the mathematical model proposed to describe distortions for visible 
light images are also appropriate for IR images [25]. As expected, distortion amplitudes are higher near the borders of the images. The values can reach 2.4 pixels, which is equivalent to $144 \mu \mathrm{m}$ in terms of physical dimensions. The distortion center coordinates are 196 and 222 pixels along $\mathrm{X}$ and $\mathrm{Y}$ directions, respectively. The radial distortion amplitude is equal to 3.7 pixels (considering a characteristic length [12] being the width of the analyzed ROI, namely 590 pixels) and the prismatic parameters are evaluated as 0.4 and -1.7 pixels. These levels are significant if an IR camera is to be used as measuring device.

(a)

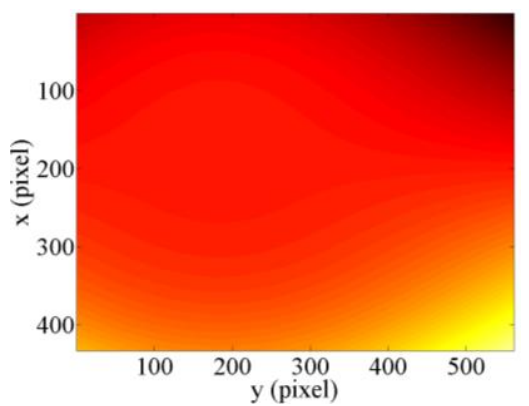

(b)

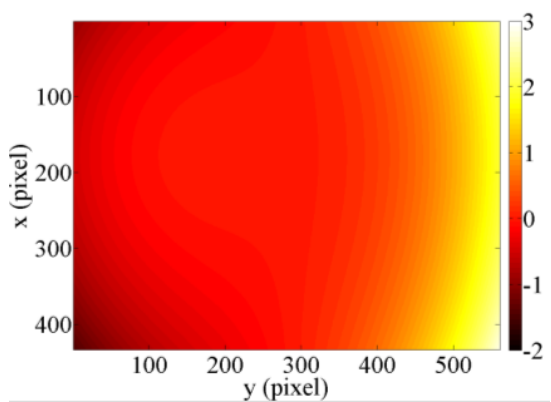

Figure 17: Distortion fields expressed in pixels along (a) $y$ (horizontal) and (b) $x$ (vertical) axes

\section{Conclusions and Perspectives}

It has been proposed to use an artificial reference to evaluate distortions of an IR camera. Such type of approach requires the gray levels to be adjusted when this picture is registered with experimentally acquired images. A new method is proposed to relax the brightness conservation usually considered in global DIC approaches. Moreover the method also accounts for blur. The performance of the I-DIC code has been determined via artificial test cases and the analysis of an IR image.

The test cases have shown the importance of considering spatially modulated gray level and blur corrections. For instance when a sine-like gray level variation, noise 
and blur are applied, the I-DIC estimation with no gray level corrections leads to dimensionless residuals as high as $30 \%$ and can be as low as $2 \%$ when all the corrections are implemented. The former calculation yields an RMS error between the prescribed and measured distortion fields of $8.2 \times 10^{-2}$ pixel, while the latter leads to a tenfold decrease $\left(8.2 \times 10^{-3}\right.$ pixel $)$ with fields of order 2 .

I-DIC analyses have shown their robustness for different cases. In terms of gray level readjustments the code provides very good results for images shot with an IR camera (compared to numerically generated references images). The same trends are observed for the change of the dimensionless residuals when the various corrections are implemented. When no corrections are considered, very high levels are observed (i.e., $30 \%)$. They are reduced to $4 \%$ when gray level and blur corrections are applied. The distortion fields using the pinhole perspective model were probed for an IR camera. Even though the final residuals are not reaching the noise level (meaning that some model error still remains) they account for most of the distortion effects.

This work provides and validates tools so that DIC can be used for registering images of different origins [13]. This open the way to the association of images of the same scene as acquired by different imaging modalities, whereby common features can be used for registration and original features of each modality can be brought to the same referential to produce an enriched image. 


\section{REFERENCES}

[1] M. A. Sutton, J.-J. Orteu, and H. W. Schreier, Image Correlation for Shape,Motion and Deformation Measurements. Springer, 2009.

[2] A. Maynadier, M. Poncelet, K. Lavernhe-Taillard, and S. Roux, "One-shot Measurement of Thermal and Kinematic Fields: InfraRed Image Correlation (IRIC)," Exp. Mech., vol. 52, no. 3, pp. 241-255, Mar. 2012.

[3] M. A. Sutton, N. Li, D. C. Joy, A. P. Reynolds, and X. Li, "Scanning Electron Microscopy for Quantitative Small and Large Deformation Measurements Part I: SEM Imaging at Magnifications from 200 to 10,000," Exp. Mech., vol. 47, no. 6, pp. 775-787, Dec. 2007.

[4] A. Guery, F. Latourte, F. Hild, and Stéphane Roux, "Characterization of SEM speckle pattern marking and imaging distortion by digital image correlation," Meas. Sci. Technol., vol. 25 , no. 1, p. 015401, 2014.

[5] C. Li, Z. Liu, and H. Xie, "Novel scanning electron microscope bulge test technique integrated with loading function," Rev. Sci. Instrum. 851037092014.

[6] J. Neggers, J. P. M. Hoefnagels, F. Hild, S. Roux, and M. G. D. Geers, "Direct StressStrain Measurements from Bulged Membranes Using Topography Image Correlation," Exp. Mech., vol. 54, no. 5, pp. 717-727, Jun. 2014.

[7] I. Chasiotis and W. Knauss, "A new microtensile tester for the study of MEMS materials with the aid of atomic force microscopy," Exp. Mech., vol. 42, no. 1, pp. 51-57, Mar. 2002.

[8] S. W. Cho, K. Jonnalagadda, and I. Chasiotis, "Mode I and mixed mode fracture of polysilicon for MEMS," Fatigue Fract. Eng. Mater. Struct., vol. 30, no. 1, pp. 21-31, Jan. 2007.

[9] K. Han and M. Ciccotti and S. Roux, "Measuring nanoscale stress intensity factors with an atomic force microscope," EPL Europhys. Lett., vol. 89, no. 6, p. 66003, 2010.

[10] B. K. Bay, T. S. Smith, D. P. Fyhrie, and M. Saad, "Digital volume correlation: Threedimensional strain mapping using X-ray tomography," Exp. Mech., vol. 39, no. 3, pp. 217-226, Sep. 1999.

[11] S. Roux, F. Hild, P. Viot, and Dominique Bernard, "Three-dimensional image correlation from X-ray computed tomography of solid foam," Compos. Part Appl. Sci. Manuf., vol. 39, no. 8, pp. $1253-1265,2008$.

[12] J.-E. Dufour, F. Hild, and S. Roux, "Integrated digital image correlation for the evaluation and correction of optical distortions," Opt. Lasers Eng., vol. 56, no. 0, pp. 121 - 133, 2014.

[13] A. Charbal, J.-E. Dufour, F. Hild, M. Poncelet, and S. Roux, "Hybrid Stereocorrelation Using Infrared and Visible Light Cameras," Exp. Mech., submitted, 2015.

[14] C. Sutour, J.-F. Aujol, C.-A. Deledalle, and B. de Senneville, "Edge-Based Multi-modal Registration and Application for Night Vision Devices," J. Math. Imaging Vis., vol. 53, no. 2, pp. 131-150, Oct. 2015.

[15] F. Hild and S. Roux, "Digital Image Correlation," in Optical Methods for Solid Mechanics : A Full-Field Approach, Wiley-VCH, Berlin (Germany). P. Rastogi and Editor E. Hack (Edts.), 2012.

[16] P. Lava, W. V. Paepegem, S. Coppieters, I. D. Baere, Y. Wang, and D. Debruyne, "Impact of lens distortions on strain measurements obtained with 2D digital image correlation," Opt. Lasers Eng., vol. 51, no. 5, pp. 576 - 584, 2013.

[17] B. Pan, K. Qian, H. Xie and A. Asundi, "Two-dimensional digital image correlation for in-plane displacement and strain measurement: a review," Meas. Sci. Technol., vol. 20, no. 6, p. 062001, 2009.

[18] J. Zhao, D. Zhao, and Z. Zhang, "Calibration and correction of lens distortion for twodimensional digital speckle correlation measurement," Optik - Int. J. Light Electron Opt., vol. 124, no. 23, pp. $6042-6047,2013$.

[19] B. Beaubier, J.-E. Dufour, F. Hild, S. Roux, S. Lavernhe, and K. Lavernhe-Taillard, "CAD-based calibration and shape measurement with stereoDIC," Exp. Mech., vol. 54, no. 3, pp. 329-341, Mar. 2014. 
[20] R. A. Hummel, B. Kimia, and S. W. Zucker, Deblurring Gaussian Blur. Courant Institute of Mathematical Sciences. Computer Science Department, 1986.

[21] R. Szeliski, Computer Vision: Algorithms and Applications. Springer, 2010.

[22] D. C. Brown, "Decentering distortion of lenses," Photogrammetric Engineering, pp. 444 462, 1966.

[23] D. C. Brown, "Close-range camera calibration," Photogrammetric Engineering, pp. 855866, 1971.

[24] Z. Tomicevic, F. Hild, and S. Roux, "Mechanics-aided digital image correlation," J. Strain Anal. Eng. Des., vol. 48, no. 5, pp. 330-343, 2013.

[25] G. Gaussorgues, La thermographie infrarouge, 4th ed. TEC\&DOC, 1999. 


\section{List of figures}

Figure 1: (a) Acquired (with IR camera) and (b) reference images of the calibration target........ 5 Figure 2 :ROIs of the (a) numerical and (b) experimental images and (c) the gray level histogram of the whole experimental image scaled down to a 8-bit dynamic range. The arrows show the two gray levels chosen for the binarization-

Figure 3 : Residuals obtained (a) before and (b) after histogram readjustment (and no blurring kernel i.e $i=0$ ) and (c) a number of Gaussian mode $i=1$.

Figure 4: $\mathrm{L} 2$ norm of difference between the prescribed displacement field and the measured results for different settings of I-DIC (binary distorted image).

Figure 5 : Nominal DIC residual errors evolution with the different types of gray level and blurring correction (binary distorted image)

Figure 6 : (a) Distorted image biased by sine-like gray level variation along the $Y$ direction, blurring and noise and (b) its gray level profile

Figure 7 : Distortion centre position $X_{O}($ pixel) for different gray level and blurring correction functions (biased distorted image)

Figure 8: Absolute error on the radial parameter (biased distorted image) for different gray level corrections and blurring kernels

Figure 9: RMS between the prescribed displacement field and the obtained results for different settings of I-DIC (biased distorted image)

Figure 10: Nominal DIC residual errors evolution with the different types of gray level and blurring correction (biased distorted image)

Figure 11: Reference image (a) when no corrections are performed, (b) corrections using order 2 fields with no blurring kernels and (c) with blurring kernels. (d) Experimental IR picture ...... 24 Figure 12: Gray level histograms corresponding to the four pictures shown in Figure 11......... 25 Figure 13: Gray level residuals for distortion estimations of the IR camera when gray level corrections are performed with (a) constant fields, and (b) 2 blurring kernels, (c) second order fields and (d) with blurring kernels

Figure 14: Dimensionless gray level residual with different types of gray level and blurring corrections for the IR image.

Figure 15: Measured displacement fields with RT3-DIC along (a) $y$ and (b) $x$ directions, and IDIC approach with second order fields and 2 Gaussian kernels along (c) $y$ and (d) $x$ directions for the IR image.

Figure 16: RMS of the difference between the displacement fields obtained for different set of the I-DIC and the RT3 approaches (IR images)

Figure 17: Distortion fields expressed in pixels along (a) $y$ (horizontal) and (b) $x$ (vertical) axes31 